\title{
MUSICALIDAD DE LAS SALAS, REALIDAD O MITO
}

\section{(THE MUSIC OF PRISMATIC ROOMS, TRUE OR FALSE)}

Francesc Daumal Domenech, Dr. Arquitecto Prof. Titular de la ETS de Arquitectura de Barcelona. ESPAÑA

\section{RESUMEN}

Se expone una breve reflexión sobre la existencia de recintos prismáticos relacionados con los acordes musicales, a través de las tres primeras frecuencias propias o estacionarias del local. Las dimensiones de estos espacios, que pueden ir desde recintos habitacionales hasta cajas para altavoces, pueden encontrarse además entre las mejores proporciones vaticinadas tanto por los expertos acústicos como por los más eminentes tratadistas arquitectónicos.

\section{SUMMARY}

This paper brings a brief reflexion on the existence of prismatic areas related to the musical chords, through the first three frequences stationary or natural to the site. The dimensions of these areas, which can range from habitable spaces to loud-speaker boxes, can also be found among the best proportions forseen both by acoustic experts and by the most eminent architectural treatise writers.

\section{Las frecuencias estacionarias y los recintos}

Las primeras frecuencias estacionarias son aquellas que pueden formarse entre dos paneles paralelos separados por una distancia $D$, cuando ambas superficies son perfectamente reflejantes desde el punto de vista acústico.

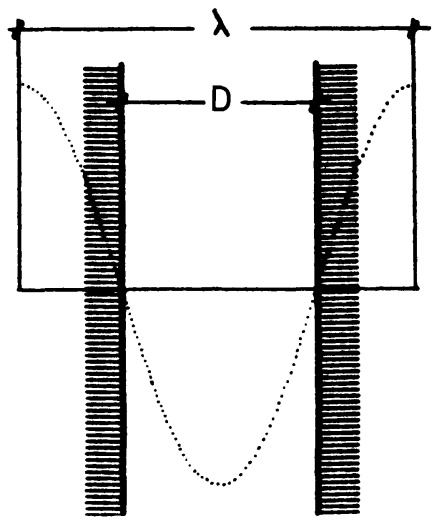

Fig. 1.- Relación entre la longitud de la onda de la primera frecuencia estacionaria y la dimensión existente entre los dos paramentos paralelos y reflejantes que la forman.
En la figura 1 podemos ver que la relación entre la distancia $D$ y la longitud de onda $\lambda$ de la primera estacionaria (la más grave), ocurre para esta longitud igual al duplo de la distancia existente entre ambos paneles.

Como sabemos, la velocidad es igual al espacio dividido por el tiempo. Si sustituimos el espacio por la longitud de una onda sonora y el tiempo por su periodo $\mathrm{T}$, nos estamos refiriendo a la velocidad del sonido $\mathrm{c}$. Siendo el período igual al inverso de la frecuencia $f$, resulta finalmente la conocida expresión:

$c=\lambda f$,

donde $\mathrm{c}$ viene dada en $\mathrm{m} / \mathrm{s}, \lambda$ en $\mathrm{m}$ y $\mathrm{f}$ en $\mathrm{Hz}$.

Sustituyendo la longitud de onda por la dimensión 2D y despejando, obtenemos la frecuencia:

$f=c / 2 D$

que no es más que la primera frecuencia estacionaria dentro de las múltiples estacionarias que se presentan en un recinto prismático (donde se forman unas 
axiles, otras tangenciales y finalmente unas oblicuas), dadas por la expresión general:

$f=(c / 2)\left\{(o / L)^{2}+(p / A)^{2}+(q / H)^{2}\right\}^{1 / 2}$,

en donde $\mathrm{L}, \mathrm{A}$ y $\mathrm{H}$ son las tres dimensiones del recinto, y $o, p$ y $q$ es una terna de valores naturales.

Con las ternas $(1,0,0),(0,1,0)$ y $(0,0,1)$, obtenemos el mismo resultado que con la expresión 2 para las primeras frecuencias estacionarias de los tres ejes en que adoptamos las dimensiones $L, A$ y $H$., por lo que continuaremos con aquella expresión más simple.

En condiciones normales de temperatura y humedad, la velocidad del sonido vale unos $344 \mathrm{~m} / \mathrm{s}$. Así, para cada dimensión obtenemos la primera frecuencia estacionaria:

$f=172 / D$,

en donde sustituiremos $D$ por el largo, ancho $o$ alto del recinto $\mathrm{L}, \mathrm{A}$ ó $\mathrm{H}$.

\section{Extensión de los recintos a estudiar}

La extensión de los recintos vendrá dada al sustituir en la fórmula 4 la gama de frecuencias audibles, que como sabemos abarca desde los 20 hasta los $20.000 \mathrm{~Hz}$.

De esta forma se observa que el local de mayor tamaño (correspondiente a su primera frecuencia estacionaria igual a $20 \mathrm{~Hz}$ ), tendrá $D=8,6$ metros.

Esta dimensión es considerada usual en los locales medianos (tipo aula pequeña, oficina de unos 50 metros cuadrados, etc.).

El límite de locales, lo tendremos cuando $D$ tenga la dimensión de un pasillo, es decir 0,8 metros, lo cual ocurre para la frecuencia estacionaria de $215 \mathrm{~Hz}$.

Con frecuencias superiores a $215 \mathrm{~Hz}$ obtenemos recintos no aptos para habitáculos, pero si para recintos tipo "bafles" de altavoces.

En este último caso, el valor límite que resulta para la frecuencia de $20.000 \mathrm{~Hz}$ es de 8,6 milímetros, muy inferior al límite de un recinto para altavoces.

\section{Extensión de las frecuencias}

Por cuanto se ha considerado de las primeras frecuencias estacionarias, vemos que en arquitectura sólo puede hablarse de las comprendidas entre 20 y $215 \mathrm{~Hz}$.

\section{Extensión musical}

Actualmente, como saben los músicos, se acostumbra a afinar los instrumentos musicales adoptando como nota fundamental el La 3 (tercera octava o La central del teclado del piano). Gracias al acuerdo de la Internacional Federation of Standardizing Associations, se adopta la frecuencia de $440 \mathrm{~Hz}$ para este La 3.

En la tabla 1 se relacionan las diferentes notas musicales con sus frecuencias correspondientes, dentro de este ámbito de estudio, comprendido entre las frecuencias de 20 y $220 \mathrm{~Hz}$, y que abarca al intervalo musical existente entre el $\mathrm{Mi}(-2)$ del pedal del órgano hasta el La (2) o segunda octava del piano.

En esta tabla se han incluido las dimensiones que resultan considerando estas frecuencias como primeras frecuencias estacionarias, y que van desde los $8,35 \mathrm{me}$ tros para dicho $\mathrm{Mi}(-2)$, hasta los 0,78 metros del La (2).

\section{Relación matemática de la música}

En la gama temperada, existe una relación matemática de las notas musicales, definida en diversas bibliografías $\{1\},\{2\}$ y $\{3\}$.

Según esta relación, cada nota tiene su frecuencia sonora igual a la de la nota anterior multiplicada por una constante.

Como se han definido 12 semitonos para la extensión de las notas de una octava, y siendo la frecuencia de la octava igual al duplo de la frecuencia fundamental, obtenemos la constante al extraer la raíz doceava del número 2, lo cual nos da como resultado el valor de 1,05946 .

En la figura 2 se han representado las diferentes no. tas musicales correspondientes a la extensión de una octava, a través de un círculo dividido en 12 partes. http://informesdelaconstruccion.revistas.csic.es 


\begin{tabular}{|c|c|c|}
\hline & $f\left(H_{2}\right)$ & $D=172 / f$ \\
\hline \multicolumn{3}{|l|}{ C } \\
\hline \multicolumn{3}{|l|}{$D$} \\
\hline$F$ & & \\
\hline$E$ & 20,60 & 8,349 \\
\hline$F$ & 21,82 & 7,880 \\
\hline & 23,12 & 7,438 \\
\hline $\mathbf{G}$ & 24,50 & 7,020 \\
\hline & 25,95 & 6,626 \\
\hline$A(-2)$ & 27,50 & 6,254 \\
\hline & 29,13 & 5,903 \\
\hline B & 30,87 & 5,572 \\
\hline C & 32,70 & 5,260 \\
\hline & 34,65 & 4,964 \\
\hline D & 36,71 & 4,685 \\
\hline & 38,89 & 4,423 \\
\hline$E$ & 41,20 & 4,174 \\
\hline$F$ & 43,65 & 3,940 \\
\hline & 46,24 & 3,720 \\
\hline $\mathbf{G}$ & 49,00 & 3,510 \\
\hline & 51,91 & 3,313 \\
\hline$A(-1)$ & 55,00 & 3,127 \\
\hline & 58,27 & 2,952 \\
\hline B & 61,73 & 2,786 \\
\hline
\end{tabular}

\begin{tabular}{|c|c|c|}
\hline & $f\left(H_{z}\right)$ & $D=172 / f$ \\
\hline$C$ & 65,40 & 2,630 \\
\hline & 69,29 & 2,482 \\
\hline$D$ & 73,41 & 2,343 \\
\hline & 77,78 & 2,211 \\
\hline$E$ & 82,40 & 2,087 \\
\hline$F$ & 87,30 & 1,970 \\
\hline & 92,49 & 1,860 \\
\hline $\mathbf{G}$ & 97,99 & 1,755 \\
\hline & 103,82 & 1,656 \\
\hline$A(1)$ & 110,00 & 1,563 \\
\hline & 116,54 & 1,476 \\
\hline B & 123,47 & 1,393 \\
\hline C & 130,81 & 1,315 \\
\hline & 138,59 & 1,241 \\
\hline D & 146,83 & 1,171 \\
\hline & 155,56 & 1,105 \\
\hline$E$ & 164,81 & 1,043 \\
\hline$F$ & 174,61 & 0,985 \\
\hline & 184,99 & 0,930 \\
\hline$G$ & 195,99 & 0,877 \\
\hline & 207,64 & 0,828 \\
\hline$A(2)$ & 220,00 & 0,782 \\
\hline & & \\
\hline
\end{tabular}

Tabla 1.-Representación de las cuatro octavas del teclado del piano y órgano, con la correspondencia entre las distintas notas musicales, sus frecuencias y las distancias para la primera estacionaria.

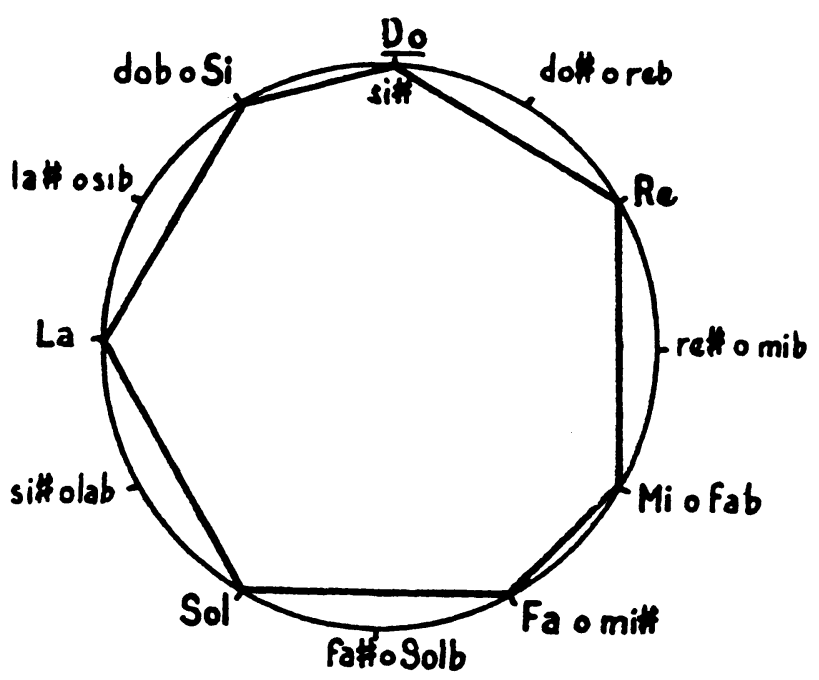

Fig. 2.-Los doce intervalos iguales de la escala temperada musical comprendidos en una octava. 


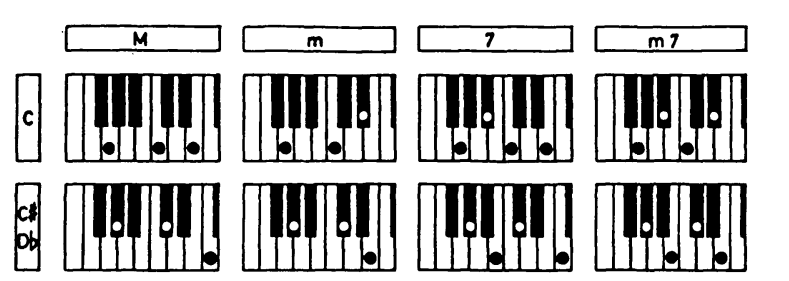

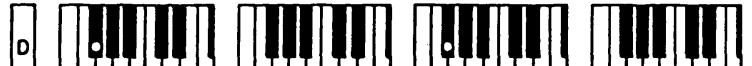

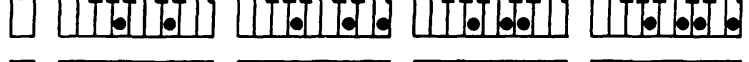

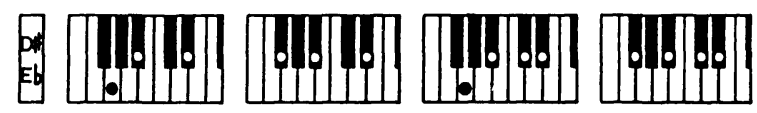

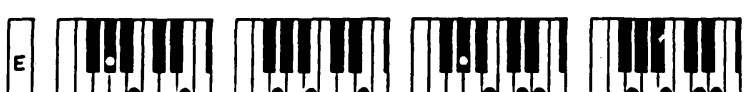

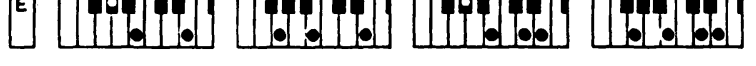

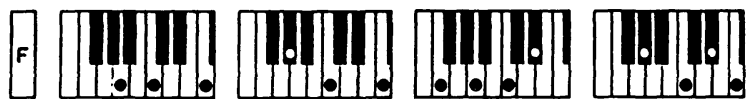

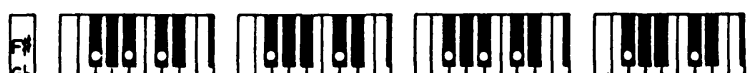

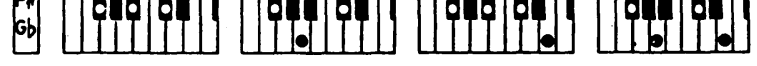

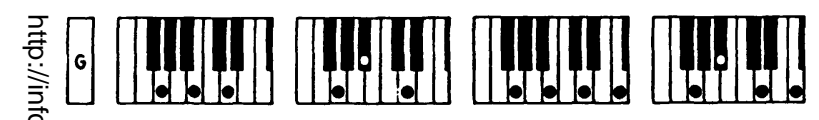
赵

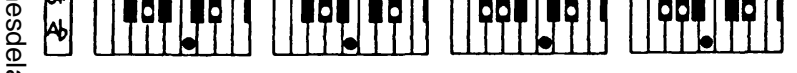

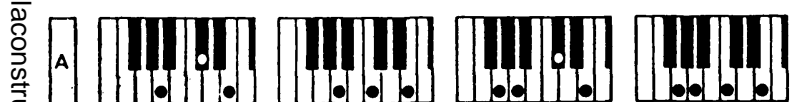
กั.

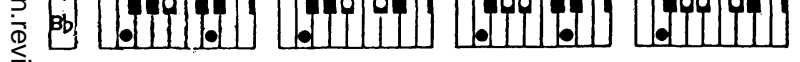

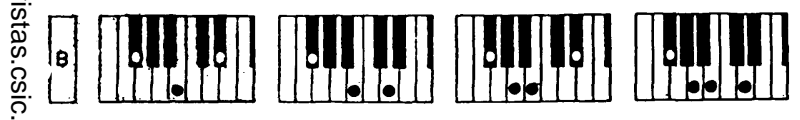

${ }_{m 7^{5}} \square 6 \square \square$

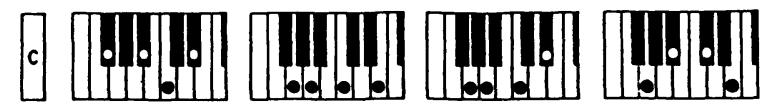

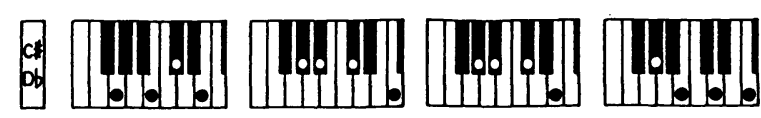

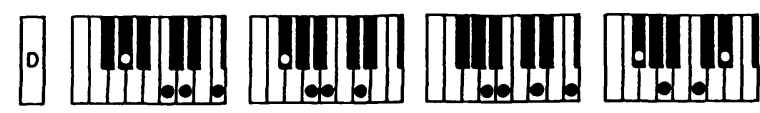

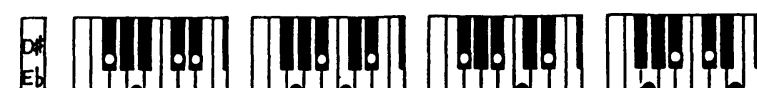

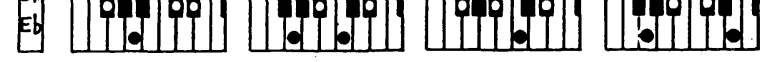

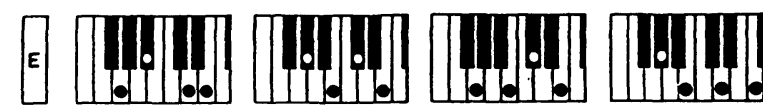

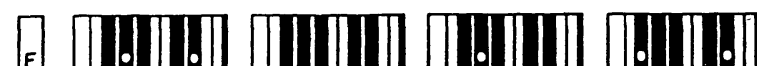

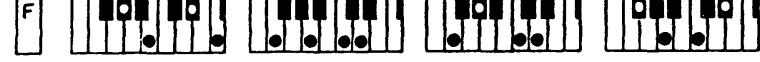

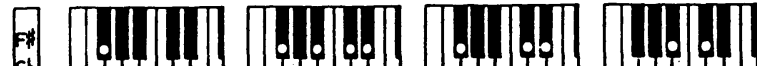

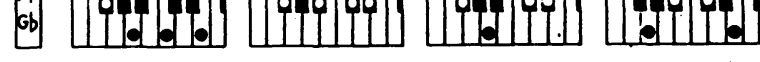

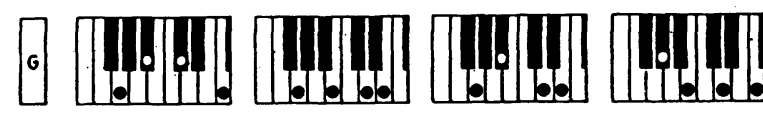

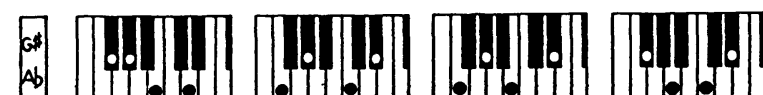

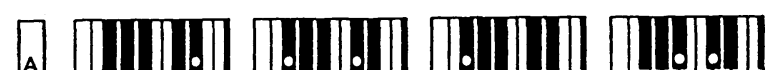

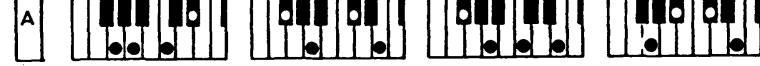

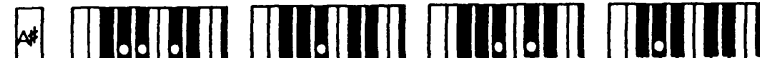

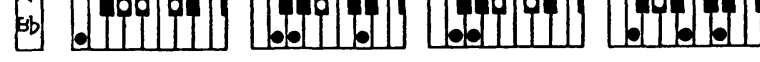

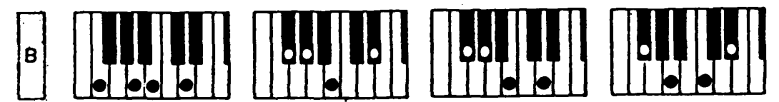

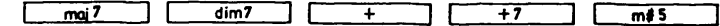

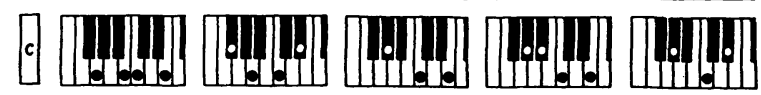

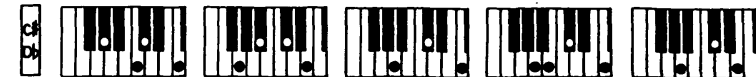

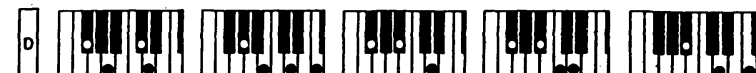

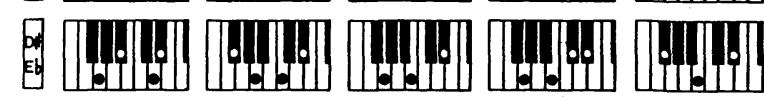

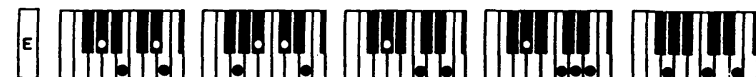

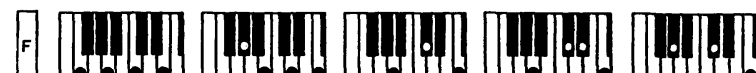
a

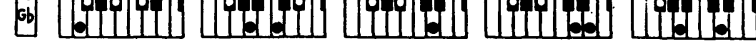

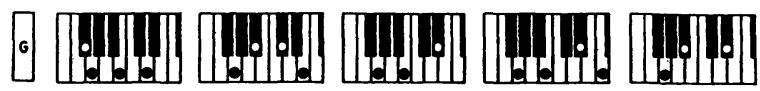

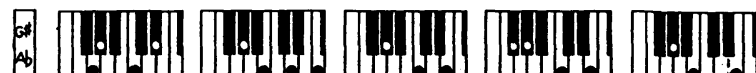

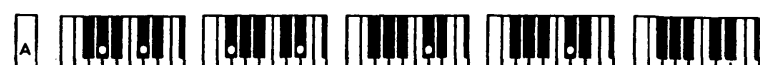
toll

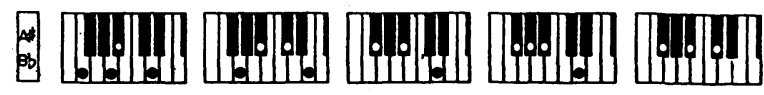

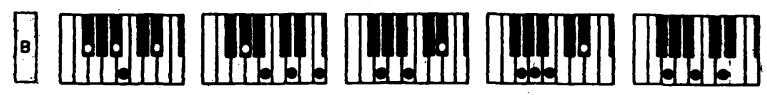




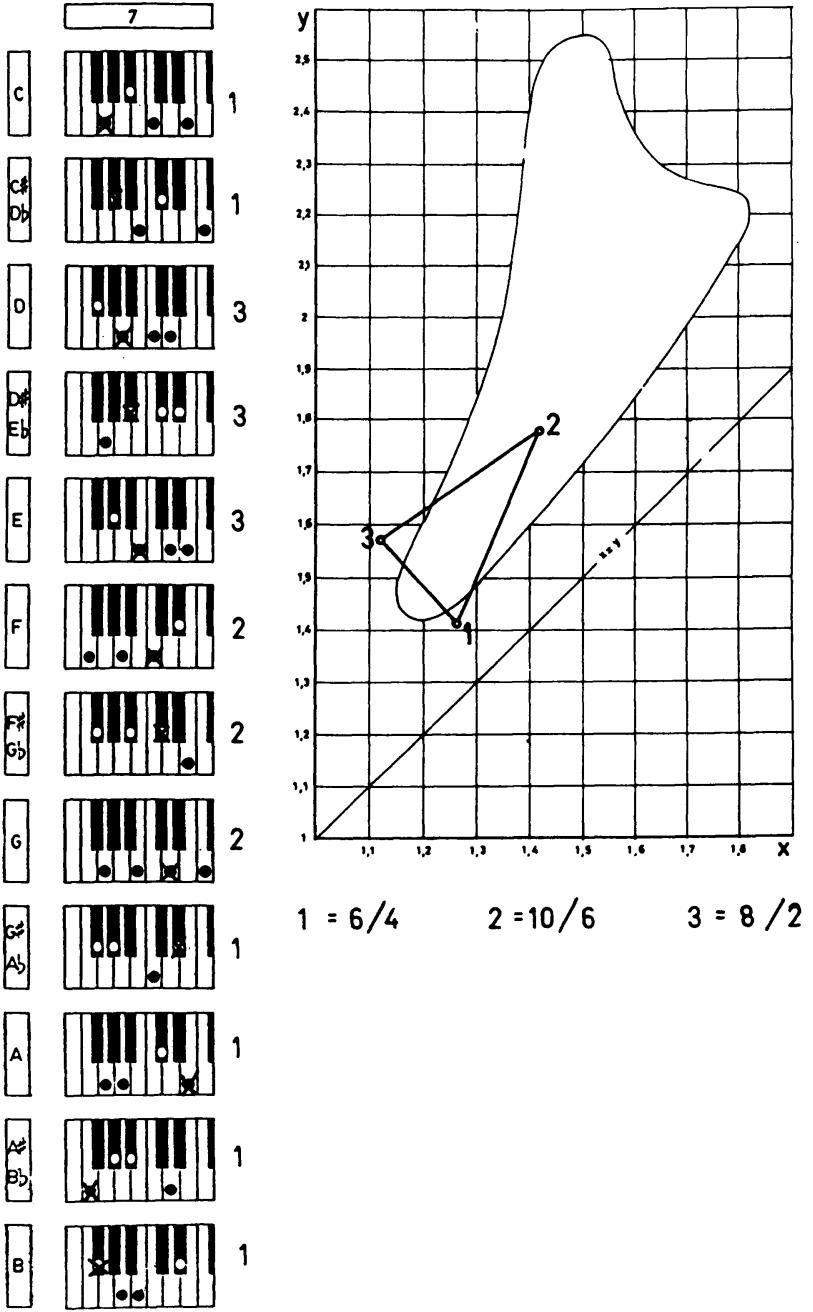

Fig. 4.-Las tres distintas posiciones del acorde de séptima (véase el número que figura a la derecha de los acordes), y las proporciones $Y=$ Largo/Alto, $X=$ Ancho/Alto, resultantes para las salas acordadas con el mismo (a la derecha).
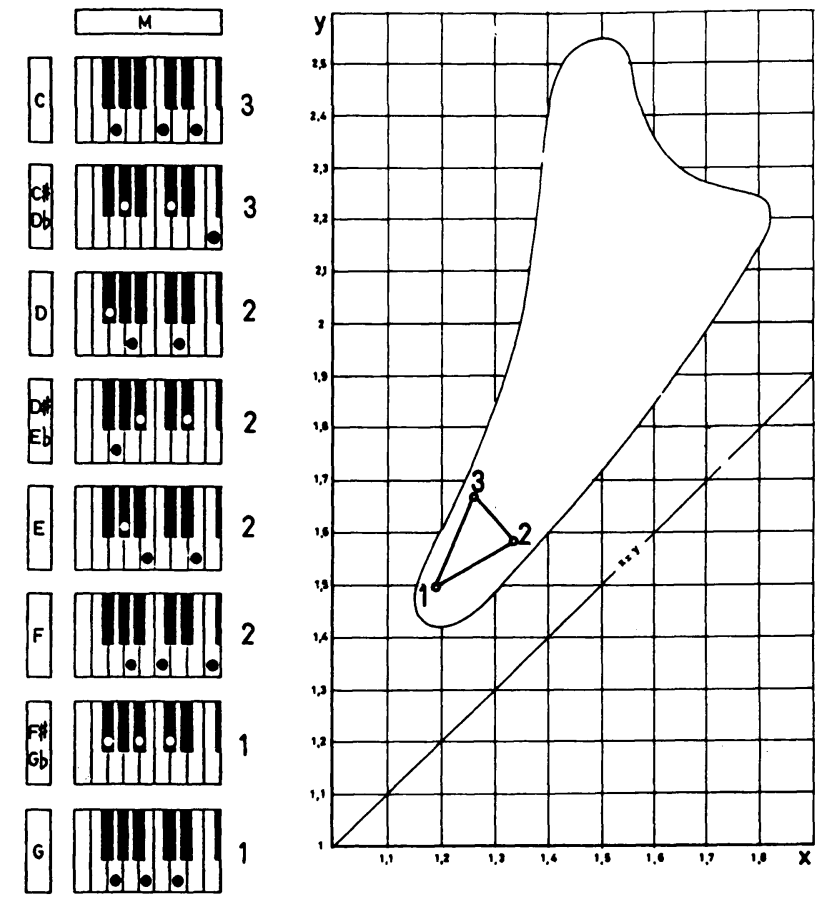

(a)

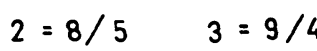

A IIIII]! 1

IIIIIII

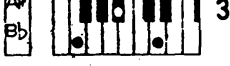

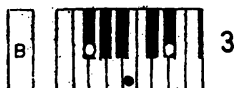

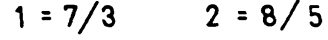

Fig. 5.-Lo mismo que la figura 4 para el acorde mayor. Obsérvese que las tres proporciones resultantes se hallan dentro del gráfico de Bolt. 


\section{Proporción de los locales}

Como hemos visto, la dimensión existente entre dos paredes paralelas está relacionada con su primera frecuencia estacionaria a través de la fórmula 4, por lo que las dimensiones $\mathrm{L}, \mathrm{A}$ y $\mathrm{H}$ de un local, se hallarán al sustituir $f$ por $\mathrm{fL}, \mathrm{fA}$ y $\mathrm{fH}$, respectivamente, de acuerdo con:

$$
\begin{aligned}
& L=172 / f L \\
& A=172 / f A \\
& H=172 / f H
\end{aligned}
$$

Podemos generalizar que en un local normal, la mayor dimensión es $\mathrm{L}$, la intermedia $\mathrm{A}$, y la menor es $\mathrm{H}$. En este caso, dividiendo las dimensiones por la menor, resultan las proporciones de un prisma unitario definido por un largo $Y$, un ancho $X, y$ una altura 1 , de acuerdo con:

$Y=L / H=f L / f H$

$X=A / H=f A / f H$

, adimensionales

Podemos ahora representar las distintas salas en un diagrama plano $\mathrm{X}, \mathrm{Y}$.

\section{Proporciones acordadas de los locales}

Busquemos ahora las proporciones de los locales que se encuentran en relación con los acordes musicales de tres notas (en el caso de acordes de cuatro notas, miraremos de anular en lo posible la que no sea significativa).

La figura 3 nos proporciona todos los acordes principales que pueden formarse para las distintas notas musicales.

Si, por ejemplo, adoptamos el acorde de La 7, vemos en la tabla 1 que con el La (0), el Do \# (1), y el Sol (1), le corresponde las siguientes frecuencias:

La $(0)=55 \mathrm{~Hz}$.

Do \# $(1)=69,29 \mathrm{~Hz}$.

Sol $(1)=97,99 \mathrm{~Hz}$.

Poniendo estas frecuencias en relación con la última (gracias a la constante 1,05946) resulta:

La $(0)=97,99 /(1,05946)^{10}$

Do\# $(1)=97,99 /(1,05946)^{6}$

(c) Consejo Superior de Investigaciones Científicas

Licencia Creative Commons 3.0 España (by-nc)
De las que resultan las proporciones:

$Y=(1,05946)^{10}=1,7817$

$X=(1,05946)^{6}=1,4141$

En la figura 2 vemos que estos exponentes a los que elevamos el valor 1,05946 , son iguales a los intervalos de los semitonos existentes entre el La y el Do \# por un lado, o entre el La y el Sol por otro.

Análogamente, se observa este hecho en el teclado del piano.

Pero resulta que este acorde puede también formarse con el Do \# (1), el Sol (1) y el La (1), o también con el Sol (1), La (1) y Do \# (2) (posición señalada como 1 en la figura 4), a los que les corresponden unas proporciones:

$Y=(1,05946)^{8}=1,5873$

$X=(1,05946)^{2}=1,1224$

y también:

$Y=(1,05946)^{6}=1,4141$

$X=(1,05946)^{4}=1,2599$

Asi pues, un acorde realizado en sus posiciones normales, produce tres prismas unitarios de proporciones distintas entre sí, pero que se mantienen para cualesquiera de las notas musicales sobre las que realizamos el acorde (ver Fig. 4).

El triángulo de prismas unitarios que puede formarse para este acorde, se ha representado en esta figura 4, en la que también se ha señalado el área óptima de Bolt \{4\}.

Por la misma razón, vemos que todos los acordes mayores (Fig. 5), nos determinan tres prismas unitarios distintos, pero coincidentes para cualquier nota en la que realizamos el acorde.

De esta forma, en la figura 6 podemos ir definiendo estos triángulos de proporciones unitarias para cualquiera de los acordes en que nos basamos.

Sólamente existe un acorde en el que los tres prismas son coincidentes. Se trata del acorde aumentada + , y ello es debido a que los intervalos existentes entre las notas que lo forman son el 8 y el 4 (ver Fig. 7). 


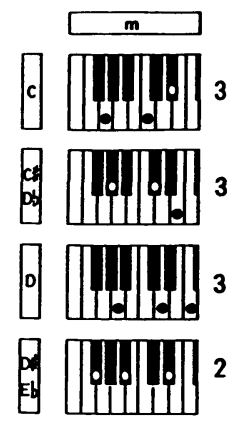

$E \prod 2$

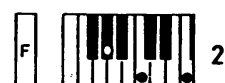
क

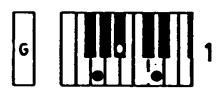
$\left.a_{0}\right)_{10}$

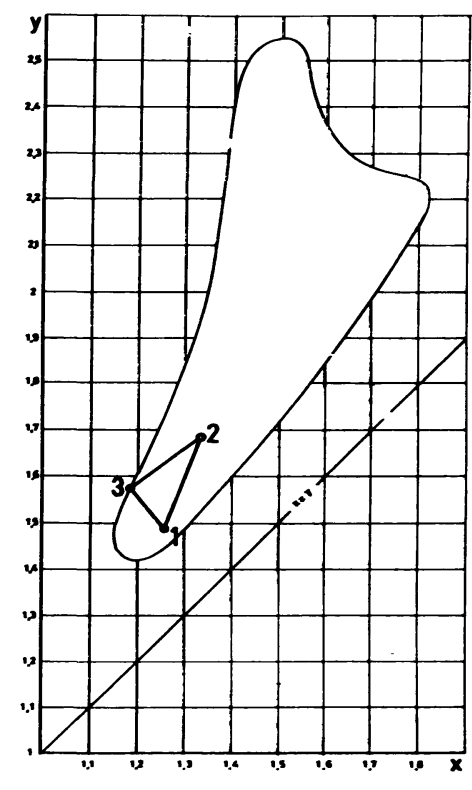

A $\prod_{b} b_{0} 1$

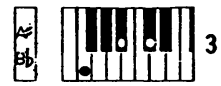

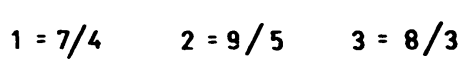

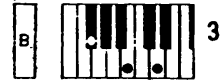

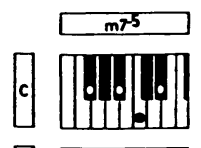

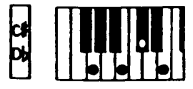

o U10

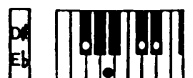

$E$ E 10.1006

F 1.1.d. d.

ab Widede

G Mbld

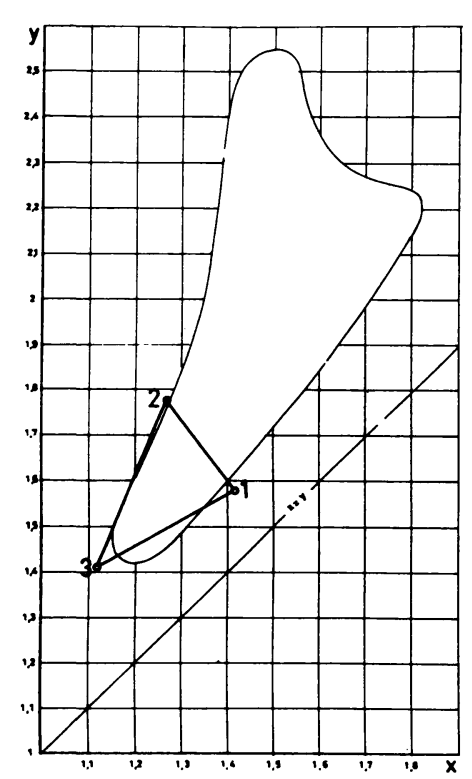

a $a$. I.

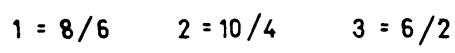

$A \longrightarrow\left\|_{b .0}\right\|_{0}$

(n) dodold

c

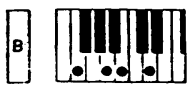

(c) Consejo Superior de Investigaciones Científicas Licencia Creative Commons 3.0 España (by-nc)
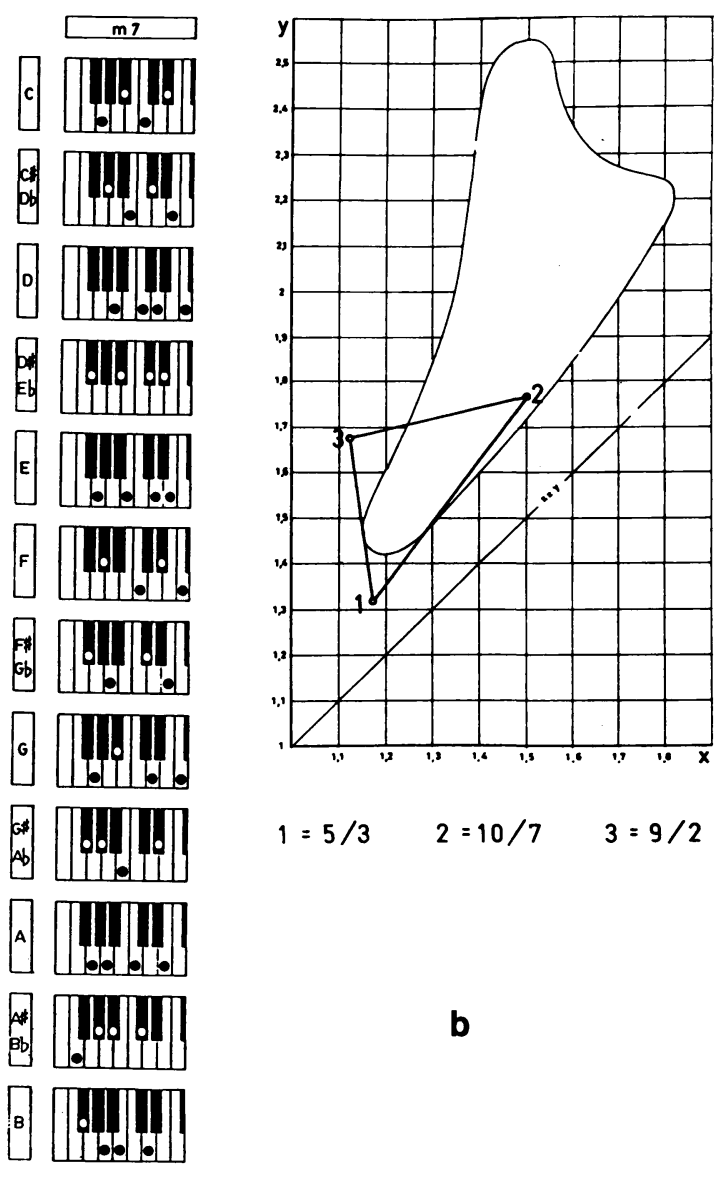

c) $\frac{6}{\prod_{0.0}}$

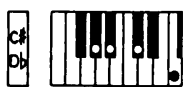

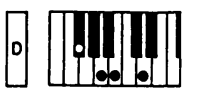

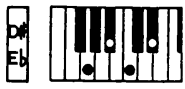

E M.d.d.d.

F M.1.0

ab To.do

$6 \prod_{60.600}$

$A b$.

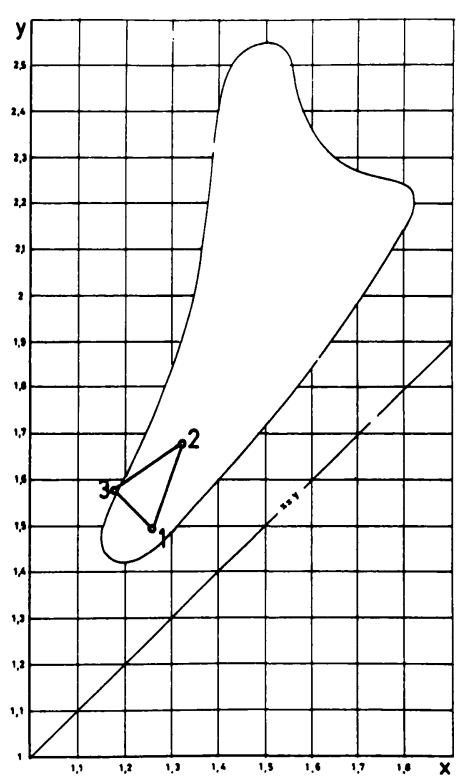

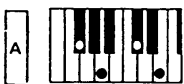

(10.0.

b
อ. 

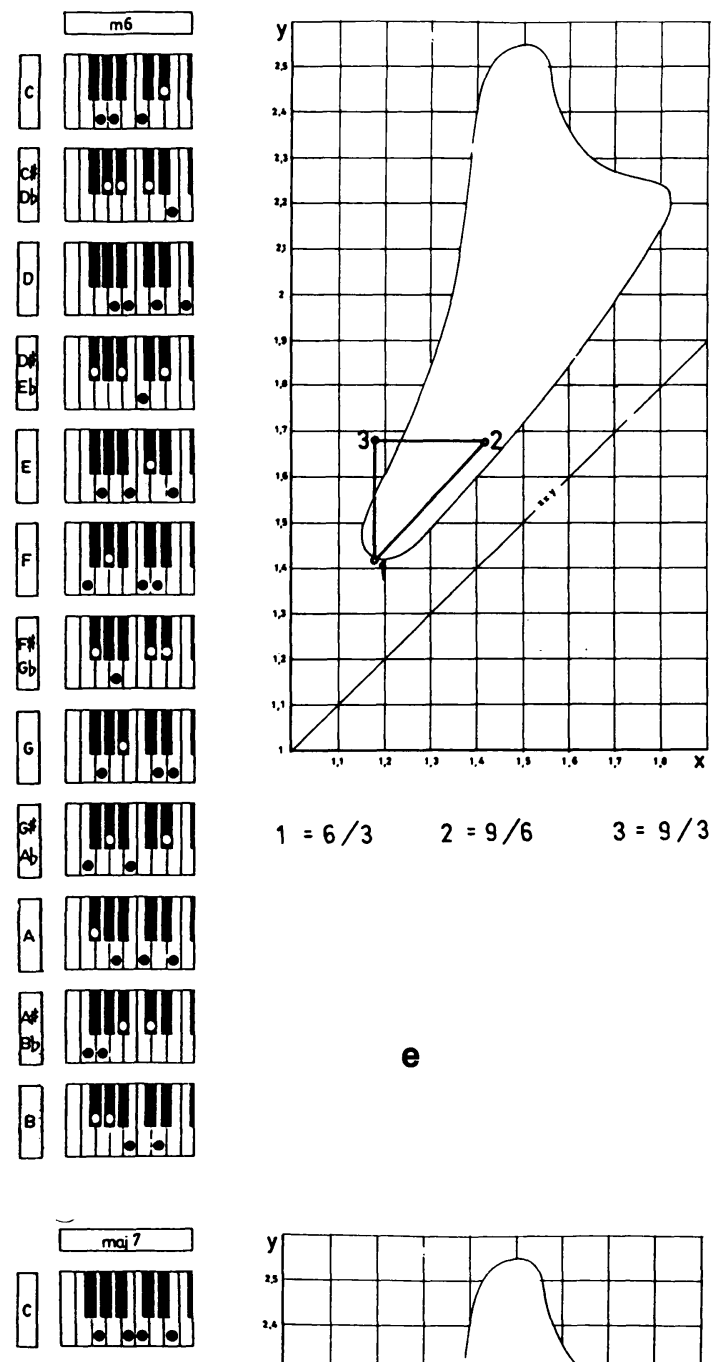

ch Mond

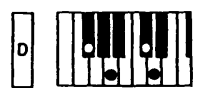

b.

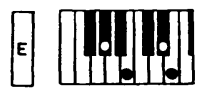

日)

ab a d

G

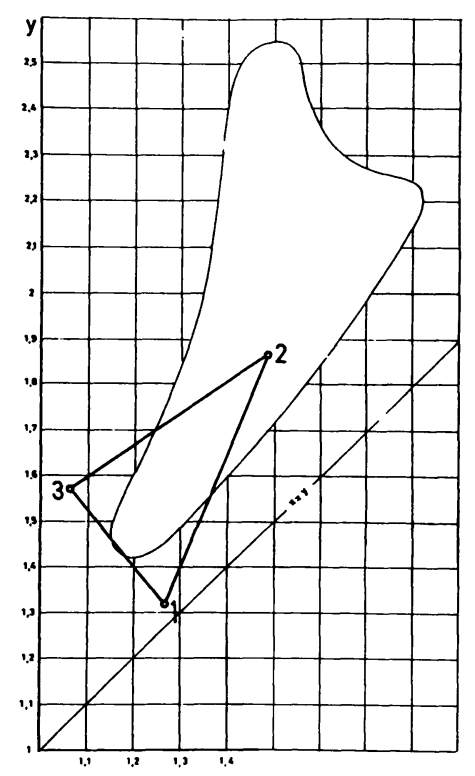

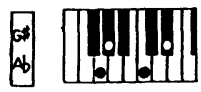

$1=5 / 4 \quad 2=11 / 7 \quad 3=8 / 1$

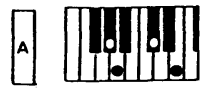

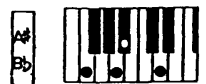

B Toldo

$\mathbf{g}$

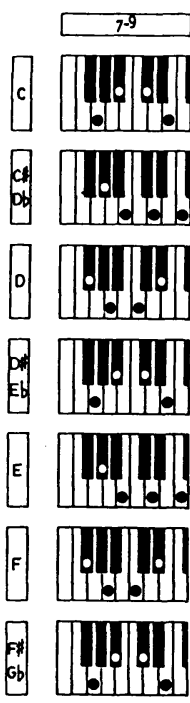

6 That

$a b)$

a $1 \mathrm{ll|l|l|l}$

周

0. [|⿴囗十)

$\operatorname{dim} 7$

c)

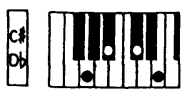

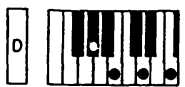

b.

E

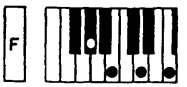

ab 1000

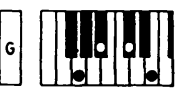

ab 4 (10)

A 10.100

Bb

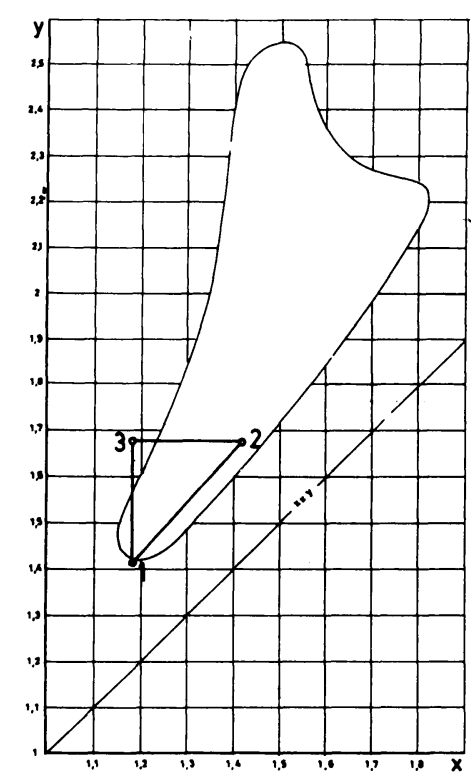

$1=6 / 3 \quad 2=9 / 6 \quad 3=9 / 3$

f

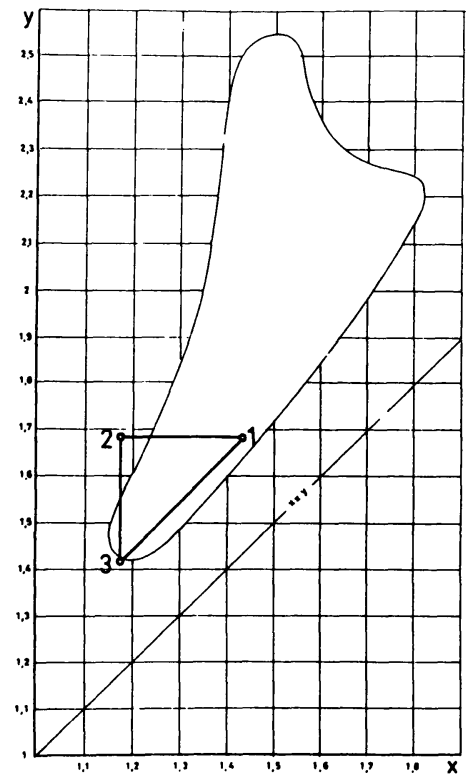

$1=9 / 6 \quad 2=9 / 3 \quad 3=6 / 3$

h 

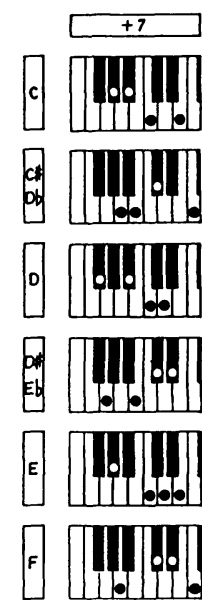

an $a b$.

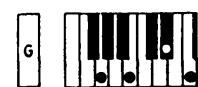

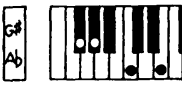

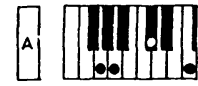

周

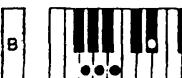

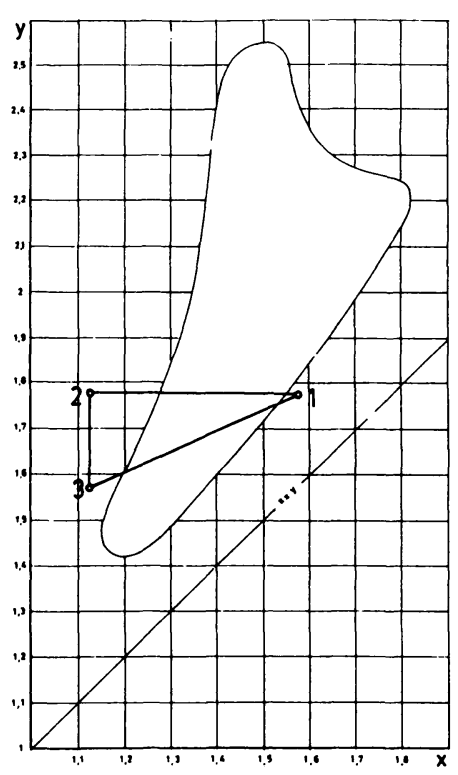

$$
1=10 / 8 \quad 2=10 / 2 \quad 3=8 / 2
$$
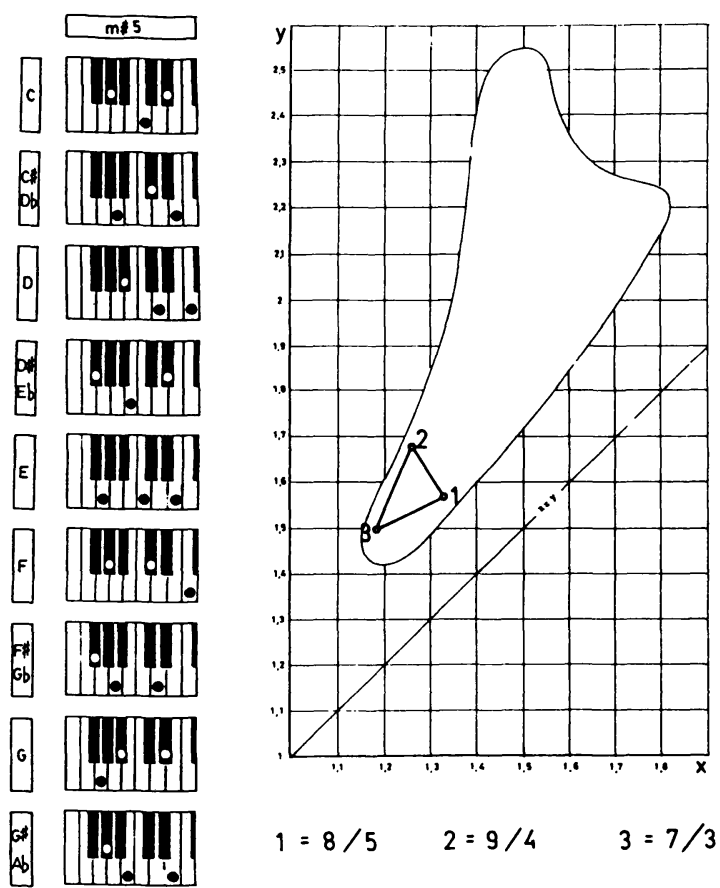

a M
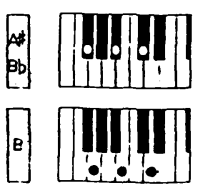

Fig. 6. - Lo mismo que la figura 4 para los acordes $m, m 7, m 7-5,6, m 6,7-9, \operatorname{maj7}, \operatorname{dim} 7,+7$, y $m \# 5$. 

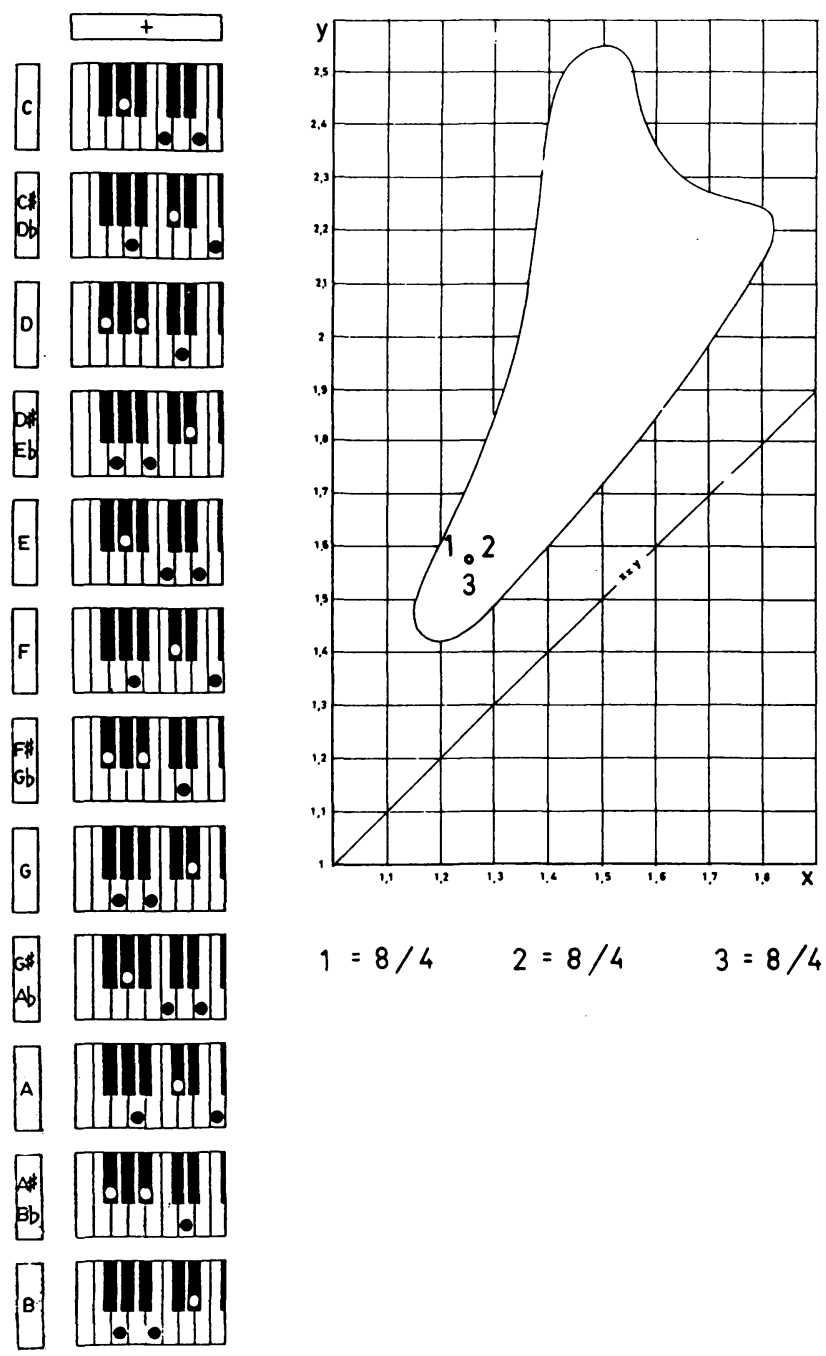

$$
1=8 / 4 \quad 2=8 / 4 \quad 3=8 / 4
$$

Fig. 7. - Lo mismo que la figura 4 para el acorde +. Obsérvese que en este caso las tres posiciones coinciden, por lo que este acorde sólo presenta una única posición en el gráfico de proporciones de los locales acordados de la derecha.

\section{Adecuación acústico-arquitectónica de los locales acordados}

En la figura 8 representamos ahora todos los triángulos juntos, observando que se hallan todos ellos dispuestos respecto al baricentro constituido por el acorde aumentada + y que, en general, ocupan unas posiciones de proporciones óptimas según Bolt.

En otros trabajos anteriores, $\{5\}$ y $\{6\}$, hemos establecido que las proporciones de las salas prismáticas de planta rectangular, han sido siempre objeto de especial atención en casi todos los tratados de arquitectura.

Desde Vitruvio hasta Bails, el objetivo de las recomendaciones, por lo que a estas proporciones se refiere,

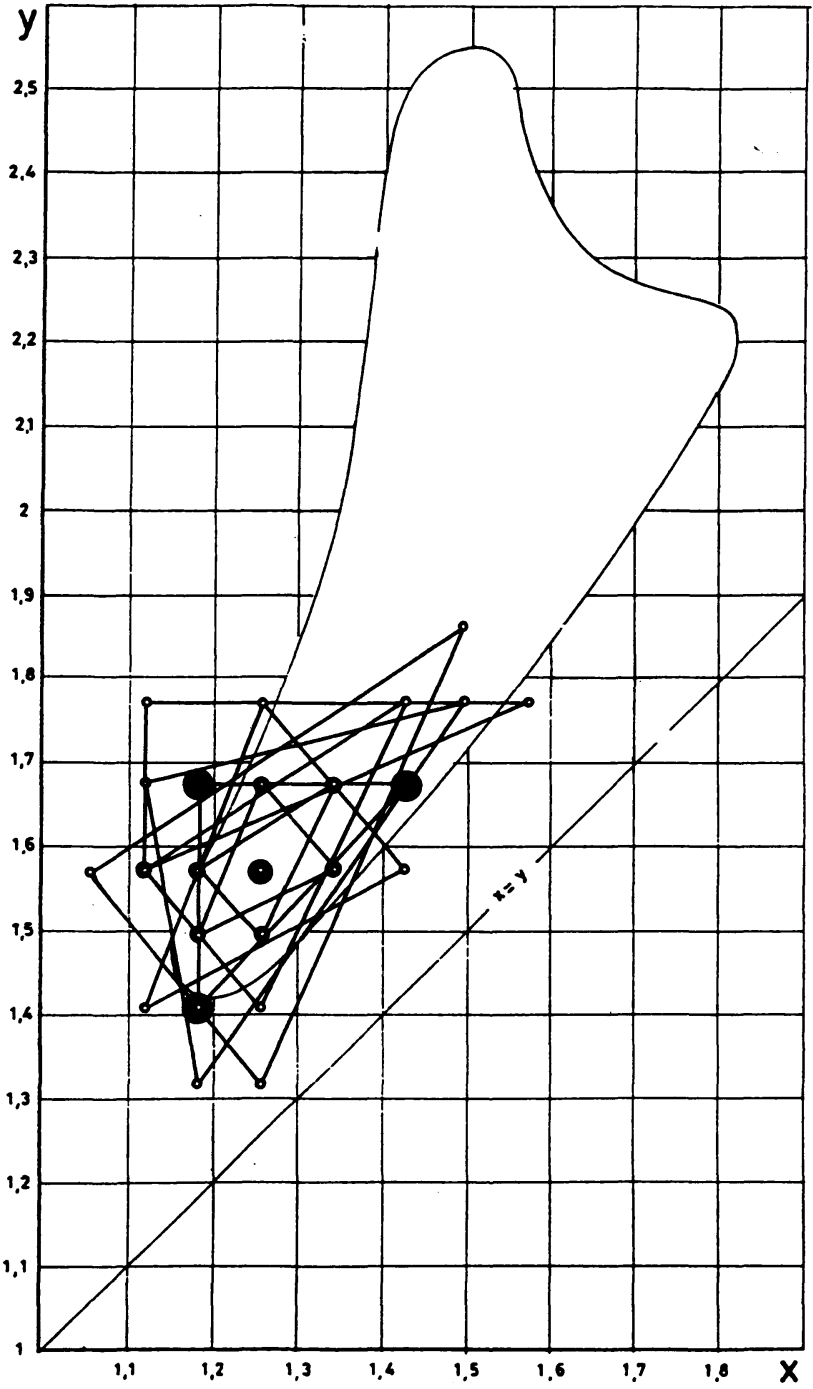

Fig. 8. - Todas las proporciones posibles de los acordes antes estudiados. Obsérvese que, curiosamente, todos los triángulos de acordes se hallan centrados respecto la posición del acorde + , lo que nos da idea de la extraordinaria relación matemática existente en la música.

se ha dedicado obviamente más a fines estéticos, que no a considerar las cualidades acústicas que estas proporciones conferían a las salas.

Como sabemos, mediante el estudio del reparto de frecuencias estacionarias definido por Bolt a mediados de este siglo, se estableció científicamente el ámbito de proporciones más aconsejable en salas pequeñas y medianas, a fin de lograr un equiespaciamiento entre las veinticinco primeras frecuencias propias de un local prismático $\{4\}$.

Ello permitió evidenciar la idoneidad o no de las "reglas de oro" propuestas por los tratadistas, tanto arquitectónicos como acústicos, para este tipo de salas (ver Figs. 9 y 10). 


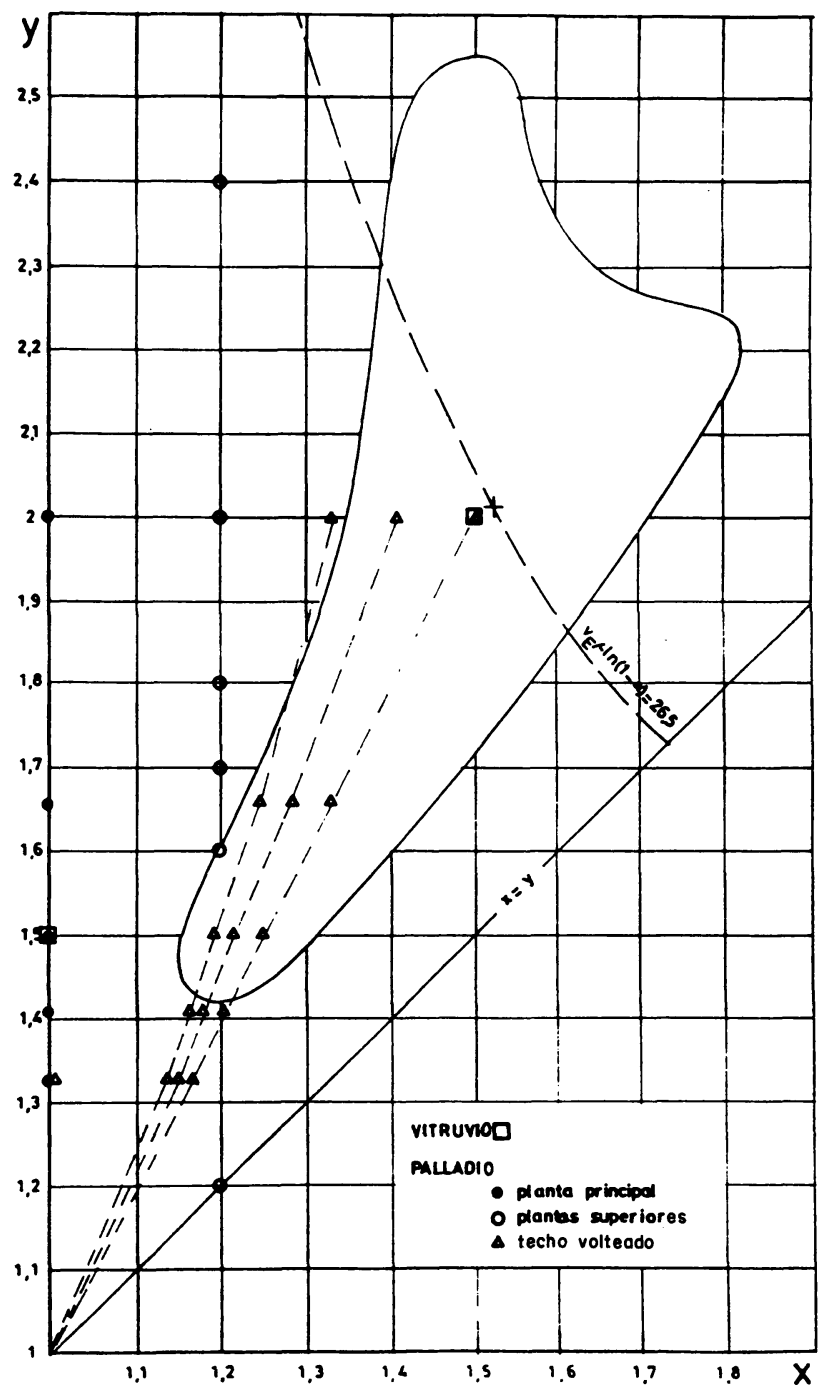

a

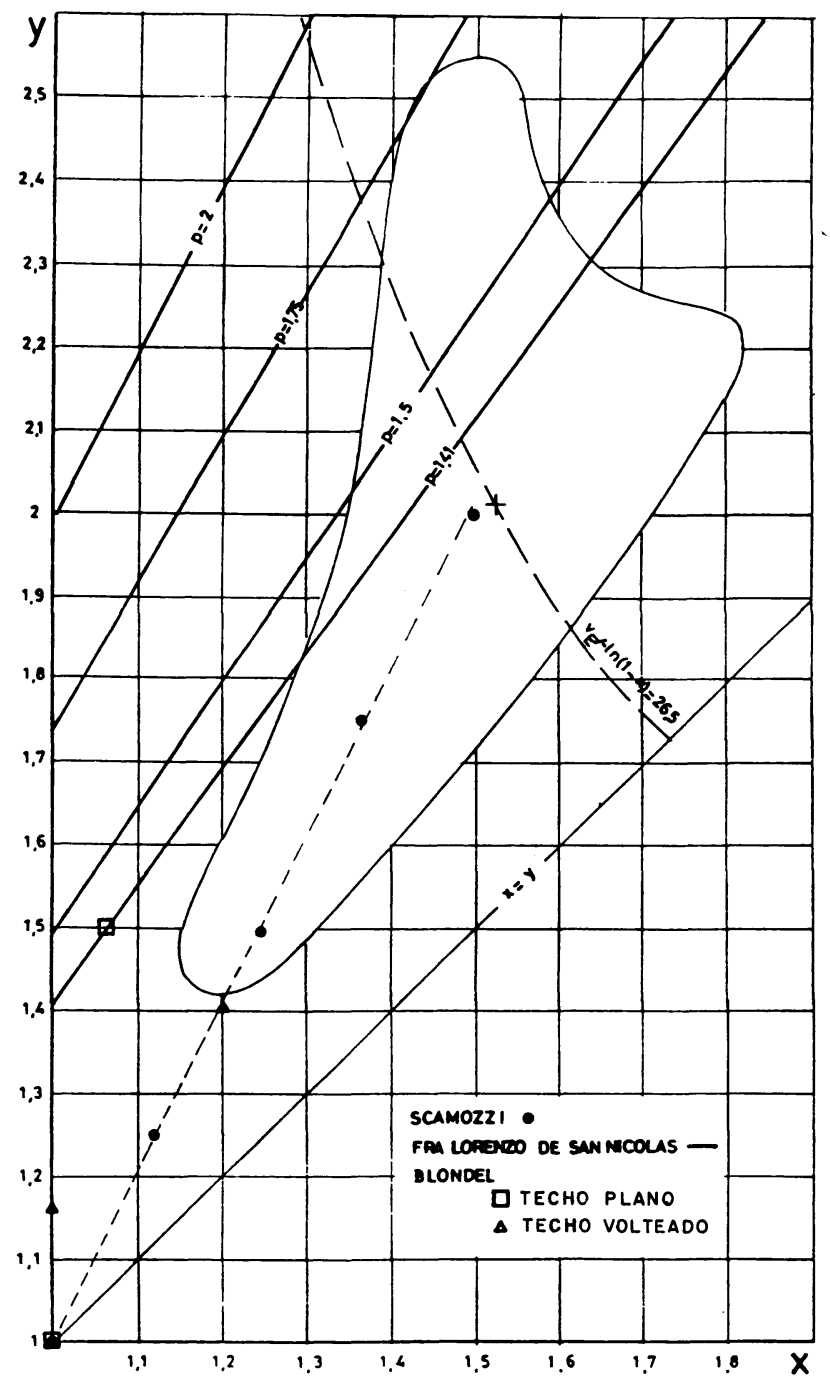

b

Fig. 9.-Las proporciones unitarias definidas por los arquitectos. a) Vitrubio y Paladio. b) Scamozzi, Fra Lorenzo de San Nicolás, y Blondel.

\section{Conclusiones}

Vemos pues que, efectivamente en múltiples casos, existe coincidencia entre las proporciones vaticinadas como óptimas por los tratadistas arquitectónicos, los acústicos, y estas proporciones resultantes del estudio sobre salas acordadas.

Por lo que hace referencia a los acústicos, en general podemos decir que existe una diferenciación entre un gran grupo que recomienda ubicar los prismas dentro o muy próximos al área de Bolt, y otros que prefieren salir de ella, alejándose tanto más cuanto mayor es el volumen del local.

(C) Consejo Superior de Investigaciones Científicas

Licencia Creative Commons 3.0 España (by-nc)
Solamente en dos casos, Barquero y Borwick, y exclusivamente para salas grandes, se recomienda sobrepasar el grado de alargamiento $p=2$, resultado de dividir la Y por la X. El límite inferior corresponde al definido por la parte baja de la zona de Bolt, casi coincidente con el valor de $p=1,15$.

Respecto a los tratadistas arquitectónicos estudiados, se aprecia la gran identificación entre la zona de Fra Lorenzo de San Nicolás con la de los acústicos Morales y Pérez Miñana, y cierto parecido con las de Sleeper y Knudsen \& Harris. Existe además cierta similitud entre algunas proporciones Palladianas y las de Bolt. También existe esta semejanza con Pérez Miñana en el caso de locales pequeños.

http://informesdelaconstruccion.revistas.csic.es 


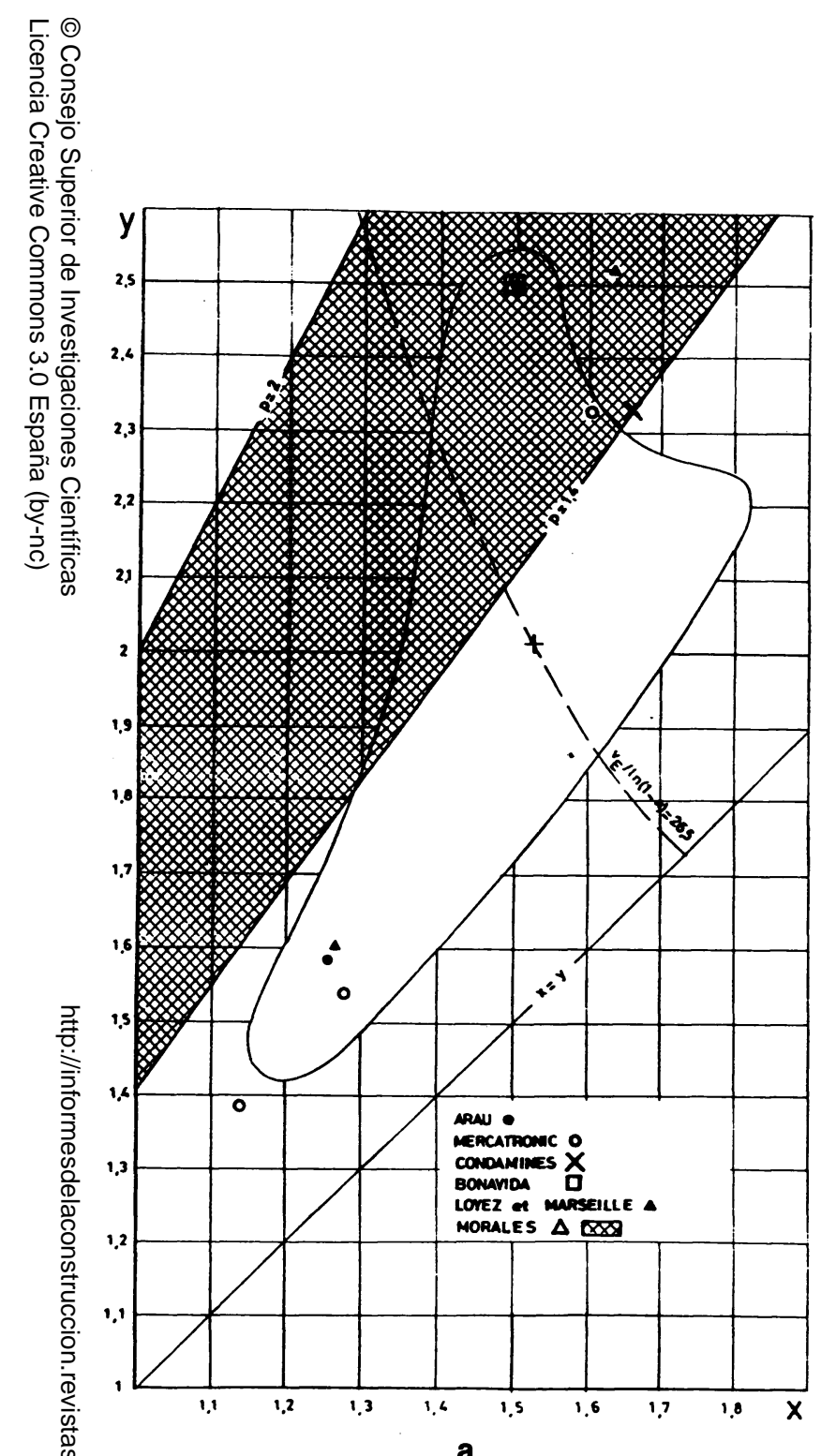

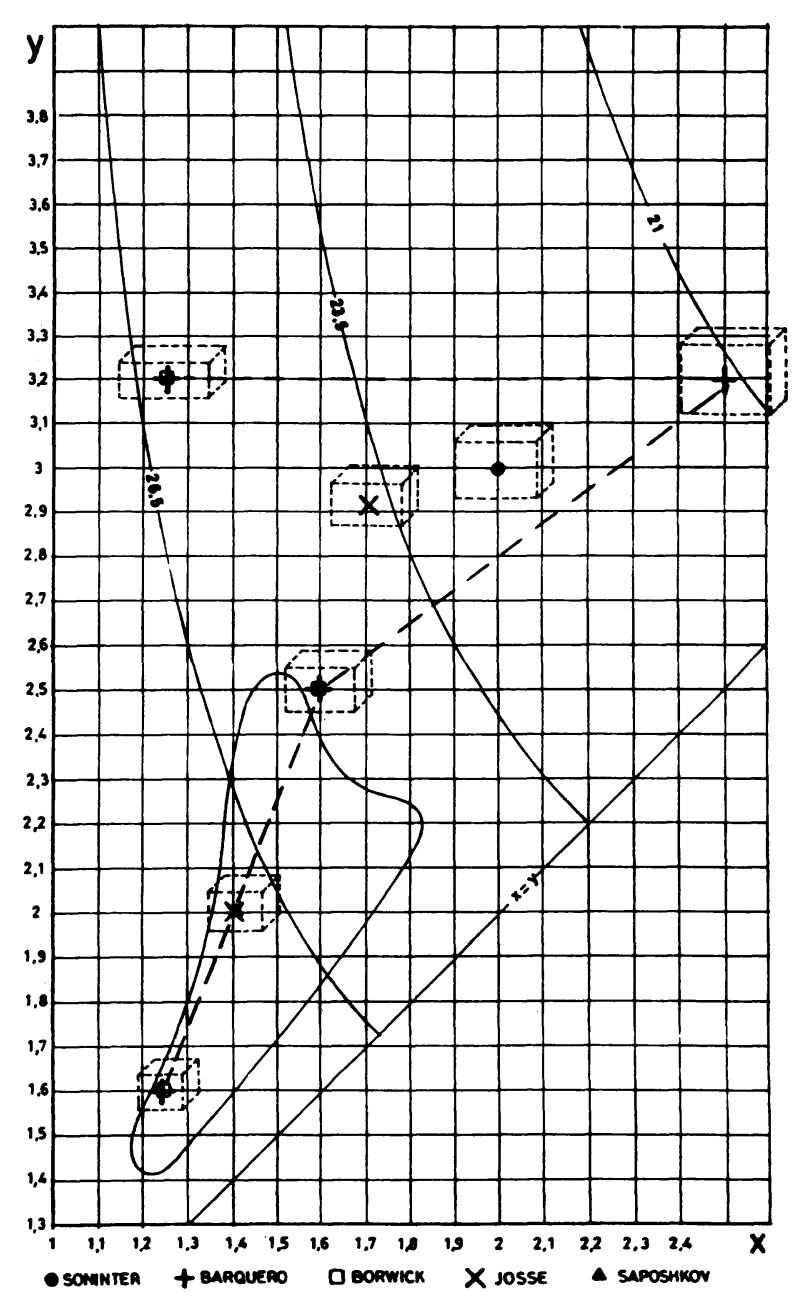

b

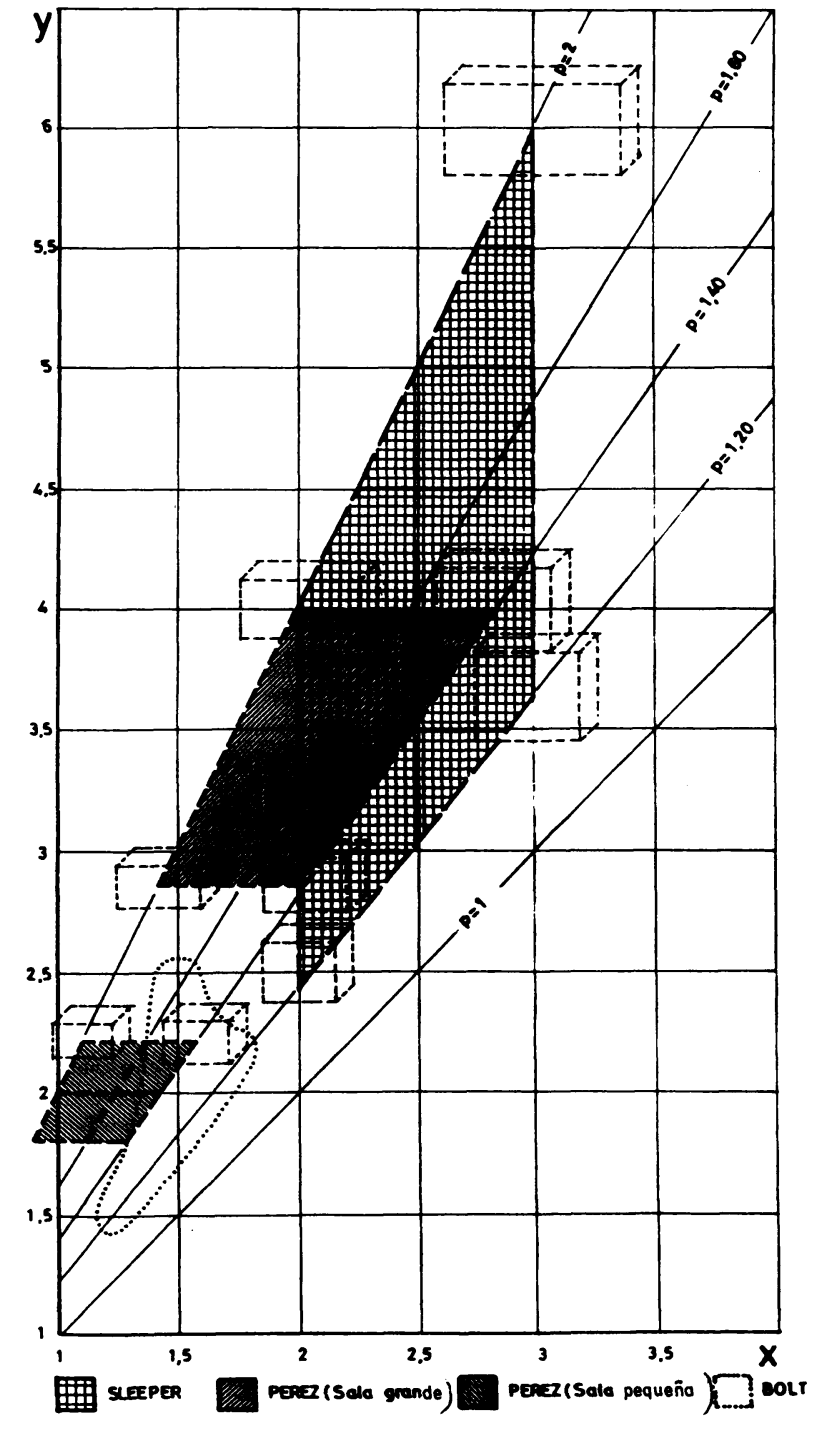

c

Fig. 10.-Las proporciones unitarias definidas por los acústicos. a) Arau, Mercatronic, Condamines, Bonavida, Loyez et Marseille y Morales. b) Soninter, Barquero, Borwick, Josse y Saposhkov. c) Sleeper, Pérez y Bolt. 
Sin embargo, del estudio sobre salas acordadas, vemos que en principio se restringe bastante la zona de acción a la parte baja del área de Bolt. Ello ocurre para los acordes "interpretados" con las posiciones normales de los dedos de una mano en el teclado del piano.

Hemos comprobado que acordes en posiciones no usuales presentan prismas unitarios localizados hacia la parte superior del área de Bolt. Sin embargo, lo inusual de su ejecución para el pianista, ha determinado que no los tengamos en cuenta en este estudio preliminar.

Se ha constatado que casi todos los acordes van originando triángulos centrados en el punto $(1,59 ; 1,26)$, que corresponde al acorde aumentada + . Vemos que los acústicos Arau y Barquero, lo recomiendan entre otros.

Este estudio, ha intentado demostrar el porqué del éxito de ciertas reglas de oro tanto arquitectónicas como acústicas, ancestralmente reconocidas por sus buenas cualidades estéticas y sonoras, y asimismo resumir las principales recomendaciones musicales existentes. El ámbito del estudio comprende desde los recintos para altavoces, pasando por pequeñas salas de audición de $\mathrm{HI}-\mathrm{FI}$, hasta llegar a los grandes auditorios.
Tal como se ha dicho, proponemos estas proporciones $(1,59 ; 1,26)$ como las que reúnen a la vez los campos Arquitectónico Acústico y Musical.

\section{BIBLIOGRAFIA}

\section{\{1\} Sir James Jeans:}

"Science and Music" (Ciencia y Música, Agora, Barcelona, 1946).

\section{(2) Daniel Blanxart:}

"Teoría Física de la Música", Bosch, Barcelona, 1958.

\section{(3) Manuel Recuero López:}

"Ingeniería Acústica", Izquierdo, S.A., Madrid, 1991.

\section{(4) José Pérez Miñana:}

"Compendio Práctico de Acústica Aplicada", Labor, Barcelona 1969.

\section{(5) Francesc Daumal:}

"L'Ambient Acústic i el Disseny Arquitectònic", Tesis doctoral, ETSAB-UPC, Barcelona, 1985.

\section{(6) Francesc Daumal:}

"Comentarios Acústicos a las Reglas de Oro de los Tratadistas Arquitectónicos", Rev. de Acústica, Vol XVII, n. 1-2, PP. 13 a 19, Octubre 1986.

\section{publicación del ICCET/CSIC}

\section{ALOJAMIENTO Y TECNOLOGÍA: ¿INDUSTRIALIZACIÓN ABIERTA? Julián Salas Serrano}

La experiencia nacional en construcción industrializada en la última década, aunque no abundante, puede resultar paralizante. Como reacción, este trabajo trata de elaborar y ordenar la información que, pegado al terreno, se ha acumulado durante los sesenta.

Auscultando tendencias avanzadas en otros paises y apoyándose en nuestra realidad cotidiana, el autor de este trabajo apuesta por la industrialización, presintiendo un futuro con pocos puntos en común con lo que en general, hasta bien reciente, se ha conocido como construcción industrializada.

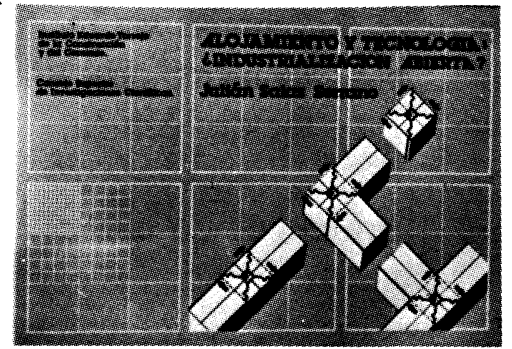

Se abre el trabajo con un prólogo del Profesor Ciribini en el cual, con rigor conceptual y desde su rica experiencia, analiza algunos de los conceptos más polémicos del fenómen de industrializacion del sector construcción. El autor recopila un conjunto de aportaciones de maestros de la arquitectura al lento proceso de evolución tecnológica y conceptual ‘ continuando con un intento de respuesta realista a la interrogante que flota en el sector nacional: ¿réquiem por la industrialización?

Se dedican otros capitulos a revisar lo realizado y a encarar el futuro inmediato: el concepto de componente, su repercusión económica, la dificultad del proyecto a base de productos industriales, la nueva vigencia embrionaria de la construcción por medio de catálogos, los conceptos de flexibilidad e intercambiabilidad desde una óptica práctica... Especial atención dedica este trabajo a la exposición de lo que podiamos llamar nuevos derroteros de la coordinación dimensional, aportando una visión actualizada y pragmática de los enfoques más implantados en Europa.

Las siempre problemáticas interrelaciones entre normativa, calidad e industria se detallan de forma documentada y realista. Finaliza el trabajo con unas reflexiones dirigidas a ámbito empresarial y un esbozo de "reglas de juego" que faciliten al subsector la salida del uimpasse" actual.

Un volumen encuadernado en rústica, de $24 \times 16,5 \mathrm{~cm}$, compuesto de 160 páginas, con 109 figuras, 19 tablas y 86 referencias bibliográficas. Madrid, junio, 1981 